\title{
ON THE SPACE OF FUNCTIONS WITHOUT DISCONTINUITIES OF THE SECOND KIND
}

\author{
L. S. GRINBLAT
}

\begin{abstract}
In this note we prove a general theorem which implies the famous proposition that the space of functions without discontinuities of the second kind, equipped with the Skorohod metric, is homeomorphic to a complete metric space.
\end{abstract}

1. Let $D[0,1]$ be the set of all functions $x(t), 0 \leqslant t \leqslant 1$, without discontinuities of the second kind. We assume that the function $x(t) \in D[0,1]$ is continuous from the right at all points $0 \leqslant t<1$ and $x(t)$ is continuous from the left at 1 . Denote by $\Lambda$ the set of all continuous and strictly increasing functions $\lambda(t), 0 \leqslant t \leqslant 1$, such that $\lambda(0)=0, \lambda(1)=1$. We shall consider $D[0,1]$ with the Skorohod metric, namely,

$$
\rho_{s}\left(x_{1}, x_{2}\right)=\inf _{\lambda \in \Lambda}\left[\sup _{t}\left|x_{1}(\lambda(t))-x_{2}(t)\right|+\sup _{t}|\lambda(t)-t|\right] .
$$

The space $D[0,1]$ is separable, but it is not a complete space. A space is said to be topologically complete if it is homeomorphic to a complete metric space. Several proofs of the topological completeness of $D[0,1]$ have been given (see, for example, [3, 3.14]). These proofs utilize the existence for $D[0,1]$ of a family of functionals $\Delta_{c}(x)(x \in D[0,1], c>0)$ which can be used to prove an analog to the Arzela-Ascoli Theorem to characterize the compact sets in $D[0,1]$. We shall prove that for an arbitrary separable metric space the existence of such an "Arzela-Ascoli type" family of functions is both necessary and sufficient to insure topological completeness.

2. THEOREM. The separable metric space $Z$ is topologically complete if and only if there exists a family $G_{c}(z)(c>0)$ of bounded continuous functions defined on $Z$ such that:

(1) $G_{c}(z) \geqslant 0$

(2) for a fixed $z$ we have $\lim _{c \rightarrow 0} G_{c}(z)=0$;

(3) $G_{c_{1}}(z) \leqslant G_{c_{2}}(z)$ if $c_{1} \leqslant c_{2}$;

(4) the closed set $K \subset Z$ is compact if and only if for any $\varepsilon>0$ there exists $\delta>0$ such that for each $z \in K$ and each $c<\delta$ we have $G_{c}(z)<\varepsilon$.

Proof. Necessity. The space $Z$ is homeomorphic to the separable complete metric space $Z^{\prime}$. Denote by $C[0,1]$ the space of continuous functions $y(t)$,

Received by the editors January 1, 1976 and, in revised form, April 29, 1976.

AMS (MOS) subject classifications (1970). Primary 54E50.

Copyright $\odot$ 1977, American Mathematical Society 
defined on $[0,1]$, with the usual metric $\rho\left(y_{1}, y_{2}\right)=\max \left|y_{1}(t)-y_{2}(t)\right|$. The Banach-Mazur Theorem asserts that a separable metric space is isometric to a subset of a space $C[0,1]$ (see $[2, \S 65]$ ). Let $Z^{\prime}$ be isometric to $Z^{\prime \prime} \subset C[0,1]$. Since $Z^{\prime}$ is a complete space, it follows that $Z^{\prime \prime}$ is a closed subset of $C[0,1]$. Consider for each $c>0$ the following functional on $Z^{\prime \prime}$ :

$$
G_{c}(y)=\min \left\{\sup _{\left|t^{\prime}-t^{\prime \prime}\right|<c}\left|y\left(t^{\prime}\right)-y\left(t^{\prime \prime}\right)\right|, 1\right\}+\min \{c \cdot \max |y(t)|, 1\} .
$$

The functionals $G_{c}(y)$ may be considered as functions on $Z: G_{c}(z)$. Obviously conditions (1)-(3) are satisfied for $G_{c}(z)$. Condition (4) is valid, according to the Arzela-Ascoli Theorem.

Sufficiency. Let $G_{c}(z)$ be a family of bounded continuous functions defined on the separable metric space $Z$, which satisfies conditions (1)-(4). By virtue of Urysohn's Theorem (see $[2, \S 58]$ ) the space $Z$ is homeomorphic to a certain subset of Hilbert space $H$. For every element $z \in Z$ let $\Phi(z)$ denote the following element of $H:\left(f_{1}(z), 2^{-1} f_{2}(z), \ldots, 2^{-n+1} f_{n}(z), \ldots\right)$, where $f_{n}$ is defined in [2, p. 128]. The set $\Phi(Z)$ is homeomorphic to $Z$. Let $G_{c}^{\prime}(z)=$ $G_{c}(z) /\left(A_{c}+1\right)$, where $A_{c}=\sup _{z} G_{c}(z)$.

For every element $z \in Z$ let $\Phi^{\prime}(z)$ denote the following element of $H$ :

$$
\left(f_{1}(z), G_{1}^{\prime}(z), 2^{-1} f_{2}(z), 2^{-1} G_{1 / 2}^{\prime}(z), \ldots, 2^{-n+1} f_{n}(z), 2^{-n+1} G_{1 / n}^{\prime}(z), \ldots\right) .
$$

The set $\Phi^{\prime}(Z)$ is homeomorphic to $Z$. The set $Q=\overline{\Phi^{\prime}(Z)}$ is the metric compactification of $Z$ such that all functions $G_{1 / n}(z)$ ( $n$ a positive integer) can be continuously extended on $Q$. Consider the closed subsets in $Q$ : $F_{m, n}=\left\{q \in Q: G_{1 / n}(q) \geqslant 1 / m\right\}$. Set $F_{m}=\cap_{n=1}^{\infty} F_{m, n}$ and $F_{\sigma}=\cup_{m=1}^{\infty} F_{m}$. Then $F_{\sigma}=Q \backslash Z$. Indeed, it is obvious that $F_{\sigma} \subset Q \backslash Z$. Suppose that there exists a point $q_{0} \in(Q \backslash Z) \backslash F_{\sigma}$. Consider the sequence of points $Z_{\infty}=\left\{z_{p}\right\}$ $\subset Z$, which converges to $q_{0}$ in the metric of $Q$. The set $Z_{\infty}$ is closed in $Z$. For any $m$ there exists $n_{1}$ such that $G_{1 / n_{1}}\left(q_{0}\right)<1 / m$. Consider the open set in $Q$ : $U=\left\{q \in Q: G_{1 / n_{1}}(q)<1 / m\right\}$. There exists a positive integer $P$ such that for $p \geqslant P$ we have $z_{p} \in U$. There exists also a positive integer $n_{2} \geqslant n_{1}$ such that $G_{1 / n_{2}}\left(z_{p}\right)<1 / m$ for $p<P$. Hence, $G_{1 / n_{2}}\left(z_{p}\right)<1 / m$ for $z_{p} \in Z_{\infty}$. This means that the sequence $Z_{\infty}$ is compact, which is a contradiction. Thus $F_{\sigma}=Q \backslash Z$. From Alexandroff's Theorem (see [1, 11.2]) it follows that $Z$ is topologically complete. Q.E.D.

3. Consider the space $D[0,1]$. The functionals $\Delta_{c}(x)$ defined in $[4, \mathrm{VI}, \S 5]$ can be altered to yield a family of continuous bounded functionals $g_{c}(x)$ satisfying the conditions of the Theorem by setting

$$
g_{c}(x)=\min \left[F_{1 / c}(x), 1\right]+\min [c \cdot \sup |x(t)|, 1]
$$

where $F_{a}$ is as defined in $[4, \mathrm{p} .430]$. This means that $D[0,1]$ is topologically complete.

'The author wishes to thank the referee for his help. 


\section{REFERENCES}

1. Gordon Thomas Whyburn, Analytic topology, Amer. Math. Soc. Colloq. Publ., vol. 28, Amer. Math. Soc., Providence, R. I., 1942. MR 4, 86.

2. Waclaw Sierpinski, General topology, Univ. of Toronto Press, Toronto, 1952. MR 14, 394.

3. Patrick Billingsley, Convergence of probability measures, Wiley, New York, 1968. MR 38 \#1718.

4. I. I. Gihman and A. V. Skorohod, The theory of stochastic processes. I, "Nauka", Moscow, 1971; English transl., Springer-Verlag, Berlin and New York, 1974. MR 49 \#6287.

Department of Mathematics, Bar-Illan University, Ramat-Gan, ISRAel 